Sharif University of Technology
Scientia Iranica
Transactions E: Industrial Engineering
hCIENTIA

\title{
Acceptance sampling plan using new truncated Weibull-X family based on run lengths of conforming items
}

\author{
M.M. Farooq ${ }^{a}$, A. Bashir, and M. Mohsin* \\ Department of Statistics, COMSATS University Islamabad, Lahore Campus, 54000, Pakistan. \\ Received 30 December 2019; received in revised form 15 November 2020; accepted 28 December 2020
}

\author{
KEYWORDS \\ Truncated Weibull-X \\ family of distributions; \\ Markov method; \\ Acceptance sampling \\ plan; \\ Conforming items; \\ Maximum likelihood \\ method.
}

\begin{abstract}
In this paper, we develop two acceptance sampling plans where the lifetimes of the products follow Weibull Exponential and Weibull Lomax distributions both of which are derived from the new truncated Weibull-X family of distributions based on run lengths of the conforming items. The model parameters are estimated using the maximum likelihood method contrary to the existing plans where authors selected arbitrary values of the parameters. The efficiency of the proposed plan is established by comparing it with the existing plan based on the average number of inspected items. A real example of failure rates of a piece of electronic equipment operating in a specific mode is presented to illustrate the proposed plans for industrial use.
\end{abstract}

(C) 2023 Sharif University of Technology. All rights reserved.

\section{Introduction}

Statistical quality control involves the implementation of various methods for monitoring and maintenance of the quality of products as well as services. "Quality is now not only an option or aim of companies but a necessity for businesses in a global market" [1]. Sampling plans are popular practicable tools used for quality affirmation and decision making, such as whether to accept or reject new lots of products. Now, it is no more an optional parameter for a business but a prioritized basic necessity to be catered for. Acceptance sampling and statistical quality control are the most important

\footnotetext{
*. Corresponding author.

E-mail addresses: muhammadfarooq@cuilahore.edu.pk (M.M.Farooq); ayeshabashir1111@gmail.com (A. Bashir); muhammadmohsin@cuilahore.edu.pk (M. Mohsin)
}

doi: $10.24200 /$ sci. 2020.55072 .4060 tools for quality assurance. Acceptance sampling plans protect both producer and customer interests. For instance, for a newly manufactured product that is ready to be dispatched into the market, sometimes it is not possible for the manufacturer to inspect all the items. In these circumstances, the use of efficient acceptance sampling plans minimizes the inspection time, costs, and efforts. These plans also include quality contracting between vendor and buyer. However, it could also convince the manufacturer to reject an acceptable lot and the buyer to accept a faulty lot. In today's fast-paced environment of manufacturing technologies and market competition, product manufacturers and suppliers require the highest standard of quality and minute fractions of non-conformities. Traditional methods and strategies for calculating fractional non-conformities are no longer helpful. In a sampling plan where time is restricted, a random sample is selected from the product batch and tested. The number of defective items is recorded within the 
specified time interval. If the number of recorded defective items is less than the minimum acceptance number then the batch is accepted. Another important factor to consider is that there are two errors associated with each sampling method regardless of the type of inspection sampling plan. Since a sample is the representation of a specific part of the products or services, there is a possibility of erroneous outcomes. The producer and customer risks are associated with these erroneous outcomes. The producer risk is the possibility of rejecting a conforming batch and the customer risk is the possibility of accepting a nonconforming batch. An efficient acceptance sampling plan is one that is designed to minimize time, effort, costs, and risks, and provide an optimum sampling size that is acceptable and affordable to both the producer and the customer.

Many trials have been conducted to develop new effective acceptance techniques to minimize the cost of inspection and save time. Bourke [2,3] proposed a sampling plan based on geometric Cumulative Sums (CUSUMs) and the run-lengths of products with successive conforming items to determine whether to perform $100 \%$ sampling inspection or phase inspection. Niaki and Fallahnezhad [4] designed an optimum acceptance plan in quality control using Bayesian inference and stochastic dynamic programming by considering cost and risk functions. Mirabi and Fallahnezhad [5] developed single-step and two-step plans using the Markovian approach and provided an acceptance policy based on the quality of inspected items. Fallahnezhad [6] determined the optimum value of thresholds based on the Markov model using the minimum angle method. Fallahnezhad and Naiki [7] proposed a Markov model to reduce costs related to machine replacement policy built on the optimal values of upper and lower limits. Balamurali et al. [8] used a double sampling plan and capability index-based variables sampling plan to propose a group acceptance plan protecting manufacturer and consumer risks. Baklizi [9] determined that the life test is trimmed at a preassigned time, based on Lomax distribution. Aslam et al. [10] used Gamma distribution to design a group acceptance plan in the life test experiment. Aslam et al. [11] suggested a decision procedure for the conforming items following the Weibull-distributed products and determined the plan parameters according to the associated risks. Chowdhury [12] designed an acceptance sampling plan under a truncated lifetime test at the pre-assigned time for generalized Weibull distribution and found the smallest sample size for the median life of the experimental unit. Shahbaz et al. [13] proposed single and double acceptance sampling plans for the power Lindley distribution by considering finite and infinite lot sizes. Al-Omari et al. [14] developed an acceptance sampling plan under the truncated lifetime test at a pre-determined time for Rama distribution and obtained the smallest sample size to ensure specified mean life for consumer risk. Mahdy and Ahmed [15] provided acceptance sampling plans for generalized inverse Weibull, skew-generalized inverse Weibull, and compound inverse Rayleigh distributions under truncated lifetime experiment to decide on the acceptance or rejection of the submitted lots. Fallahnezhad and Saredorahi [16] used the Bayesian approach to develop a new acceptance sampling plan by examining the minimum proportion of the lot with inspection errors.

Some recent contributions to the development of acceptance sampling plans using different methodologies, selecting different quality characteristics, and objective functions based on different probability distribution, are also stated. Fallahnezhad and Seifi [17] proposed a new repetitive group sampling plan based on the process capability index for variable inspection. They designed different sampling plans where the study variable follows the Bayesian approach. Fallahnezhad and Yazdi [18] introduced a sampling plan based on the conforming run-length where the consumer loss is estimated using the Taguchi loss function. They assumed two constraints for model development, the average outgoing quality limit and the lot tolerance percent defective. Rasay et al. [19] used a sequential sampling plan to truncate life test and design a repetitive group sampling plan and a double sampling plan. Fallahnezhad and Saredorahi [20] provided a variable repetitive group sampling plan based on the expected loss function. They used acceptable quality level and limiting quality level to satisfy the priorities of the producer and the requirements of the consumers according to the set criteria. Al-Omari and Al-Hadhrami [21] developed the acceptance sampling plans for time truncated life tests based on extended exponential distribution. Al-Omari [22] proposed a new acceptance sampling plan where the truncated life test follows the Sushila distribution. Aslam et al. [23] designed a group acceptance sampling plan based on neutrosophic statistics when the mean life of the product follows Weibull distribution. Singh and Buttar [24] developed a sequential sampling plan for generalized exponential distribution based on a truncated life test, taking the median life of an item as quality characteristic. AlNasser and Obeidat [25] presented a single acceptance sampling plan when the average lifetime of the product follows Tsallis $q$-exponential distribution. Divecha and Raykundaliya [26] proposed a two-stage time censoring scheme to develop three economical acceptance sampling plans by following the method of modified exponentially weighted moving average.

Several probability distributions have been extensively used in various fields like finance, economics, reliability analysis, engineering, medicine, and insur- 
ance. Probability distributions play a vital role in quality assurance as well. We see several distributions compounded with the Weibull distribution become more flexible through generalization. Luko [27] highlighted the importance of Weibull distribution to study the tenacity and strength of the material and devices in engineering. Mandouh [28] introduced the Lomax-Modified Weibull distribution and discussed its properties and Bayesian inference. Hanook et al. [29] proposed a Beta Inverse Weibull distribution generated from the logit of a Beta random variable. Cordeiro and de Castro [30] defined the Kumaraswamy-class of distributions. Ahsanuallah et al. [31] proposed a new bivariate pseudo-Weibull distribution to find the distribution of concomitants of upper record statistics. Alzaatreh et al. [32] proposed a method to create a continuous family of distributions by the technique of random variable where each generated distribution is taken as a weighted hazard function. Aljarrah et al. [33] suggested a technique similar to Alzaatreh et al. [32] based on quantile function. Rather and Rather [34] proposed k-generalized Exponential distribution which includes generalized exponential and Weibull as special cases. Mahdavi and Oliveira Silva [35] proposed a method for the expansion of continuous distribution based on truncated distribution.

The present research contributes to acceptance sampling plans based on the Weibull family of distributions by constructing Weibull Exponential (WE) and Weibull Lomax (WL) distributions following the technique of Mahdavi and Oliveira Silva [35]. The proposed distributions are used to design an acceptance sampling plan for conforming items. The proposed acceptance sampling plan and the study of Aslam et al. [11], both based on the Markovian approach and using Weibull distributions, are compared to estimate the expected average number of inspected items using optimum parameter values. The distinction of this paper is that the model parameters are estimated using the maximum likelihood method contrary to the existing plans where authors selected arbitrary values of the parameters. The successful application of the proposed plan in real-life examples supports the argument that our plan is more time and costeffective.

The rest of the paper is organized as follows: The notations are given in the subsequent section. Model development is presented in Section 2. The proposed plans based on the Weibull-X family of distributions containing Lomax and Exponential distributions are discussed in Section 3. The comparison of our plans with the existing plan is provided in Section 4. A real example of failure rates of a piece of electronic equipment to check the efficacy of our proposed plans is demonstrated in Section 5. Some concluding remarks are stated in Section 6.

\section{Model development}

In this section, the model is developed using the technique of Mahdavi and Oliveira Silva [35]. They proposed a new family of truncated TF-G distributions where $G$ is an absolutely continuous baseline distribution. The expressions for the cumulative distribution function (cdf) and the probability density function (pdf) of the TF-G family of distributions are given as:

$$
G(x)=\frac{H(F(x))-H(0)}{H(1)-H(0)},
$$

and:

$$
g(x)=\frac{h(F(x)) f(x)}{H(1)-H(0)},
$$

respectively, where $H(0)$ and $H(1)$ are the cdfs of the generator distribution evaluated at 0 and 1 while $f(x)$ and $F(x)$ represent the pdf and the cdf of the input distributions. We consider an exponential distribution and a Lomax distribution as input distributions to propose WE and WL distributions. In this paper, we take $h(t)$ as Weibull distribution with parameters $k$ and $\gamma$ having cdf and pdf as:

$$
H(t)=1-e^{-\left(\frac{t}{\gamma}\right)^{k}}
$$

and:

$$
h(t)=\frac{k}{\gamma}\left(\frac{1}{\gamma}\right)^{k-1} e^{-\left(\frac{1}{\gamma}\right)^{k-1}},
$$

respectively. So the proposed Weibull-X family of distributions has the cdf and the pdf as:

$$
G(x)=\frac{1-e^{-\left(\frac{F(x)}{\gamma}\right)^{k}}}{1-e^{-\left(\frac{1}{\gamma}\right)^{k}}}
$$

and:

$$
g(x)=\frac{k}{\gamma}\left(\frac{F(x)}{\gamma}\right)^{k-1} e^{-\left(\frac{F(x)}{\gamma}\right)^{k-1}} f(x) .
$$

\subsection{Markov method}

The Markovian approach is used to determine optimal values of the thresholds for acceptance or rejection of an experimental batch. Suppose that for an acceptance plan; $Y_{i}$ is described as the number of conforming items between two defective items with upper control limit $U$ and lower control limit $L$. In this case, the decision rule is described as:

- If $L<Y_{i}<U$ then the batch is in the transient state and the inspection continues;

- If $Y_{i} \geq U$ then the batch is in the accepted state;

- If $Y_{i} \leq L$ then the batch is in the rejected state.

State-1 $\left(L<Y_{i}<U\right)$ : The value of $Y_{i}$ is between upper and lower control limits; therefore, testing continues; 
State-2 $\left(Y_{i} \geq U\right)$ : The batch is in good condition and will be accepted;

State-3 $\left(Y_{i} \leq L\right)$ : The batch is in bad condition and will be rejected.

The probability of continuing the inspection of ' $n$ ' items is:

$$
p_{11}=P\left\{L<Y_{i}<U\right\}=\sum_{Y_{i}=L+1}^{U-1} p(1-p)^{Y_{i}} .
$$

The probability of batch acceptance is:

$$
p_{12}=P\left\{Y_{i} \geq U\right\}=\sum_{Y_{i}=\mathrm{U}}^{\infty} p(1-\mathrm{p})^{Y_{i}} .
$$

The probability of batch rejection is:

$$
p_{13}=P\left\{Y_{i} \leq L\right\}=\sum_{Y_{i}=0}^{\mathrm{L}} p(1-\mathrm{p})^{Y_{i}} .
$$

$Y_{i} \sim$ geometric distribution with parameter $p$ and its pdf is given as:

$$
P\left(Y_{i}=r\right)=p(1-p)^{r},
$$

where $r=0,1, \ldots$ The transition probability matrix of the above three states of the batch can be expressed as:

$$
P=\left[\begin{array}{ccc}
p_{11} & p_{12} & p_{13} \\
0 & 1 & 0 \\
0 & 0 & 1
\end{array}\right]
$$

Matrix $P$ in Eq. (10) is an absorbing Markov model in which State- 1 is transient while States- 2 and 3 are absorbing, i.e., once it has reached a state, it can never leave. Since State-I denotes the probability of inspecting more items $p_{11}$ is the probability of transition from State-1 to State-1 i.e., we need to inspect more items. Hence, the transition probability matrix is reorganized as:

$$
P=\left[\begin{array}{ll}
A & O \\
R & Q
\end{array}\right]
$$

where $A$ denotes identity matrix, $O$ is a matrix of zeros, $R$ is the matrix comprising of probabilities of moving from a non-absorbing state to an absorbing state and $Q$ is a square matrix comprising of transition probabilities of going from a non-absorbing state to another nonabsorbing state. This reorganization gives the following form of transition probability matrix:

$$
P=\left[\begin{array}{ccc}
1 & 0 & 0 \\
0 & 1 & 0 \\
p_{12} & p_{13} & p_{11}
\end{array}\right]
$$

The fundamental matrix $M$ can be expressed as follows [36]:

$$
M=m_{11}=(I-Q)^{-1}=\frac{1}{1-\operatorname{Pr}\left\{L<Y_{i}<U\right\}},
$$

where " $I$ " denotes the unit matrix, " $m_{11}$ " denotes the expected number of times occupying the transient State-1 before absorption occurs in the long-run. The long-run expected probability matrix $F$ can be computed as follows:

$$
F=M \times R=\left[\begin{array}{ll}
\frac{p_{12}}{1-p_{11}} & \frac{p_{13}}{1-p_{11}}
\end{array}\right] .
$$

Here, the elements $p_{12}$ and $p_{13}$ of this matrix represent the probability of acceptance and rejection of a batch respectively.

\section{Proposed plan based on Weibull-X family of distributions}

The Weibull-X family of distributions, containing Lomax and Exponential distributions, has been used for modeling lifetime data and phenomenon with monotonic failure rates. In this paper, we develop WE and WL distributions using the technique of Mahdavi and Oliveira Silva [35] and use them to propose new acceptance sampling plans. The positively and the negatively skewed density shapes of the Weibull-X family of distributions may be a starting choice for modeling monotonic failure rates.

It is assumed that the lifetime of a product follows the Weibull-X distribution given in Eq. (5) with a scale parameter greater than zero. Then the probability of failure of an item before experiment time $t_{0}$ is expressed as:

$$
p=\frac{1-\exp \left(-\left(\frac{1-\exp \left(-F\left(\frac{t_{0}}{\lambda}\right)\right)}{\gamma}\right)^{k}\right)}{1-\exp \left(-\left(\frac{1}{\gamma}\right)^{k}\right)} .
$$

Let the termination time ratio be $\frac{t_{0}}{\mu_{0}}=a$, which implies that $t_{0}=a \mu_{0}$ for the constant value of $a$. Thus, an item is categorized as a nonconforming item only when its failure occurs before the termination time. The average number of items inspected $E(I)$, for each visit to the transient state, will be the product of the mean of the geometric distribution $(1 / p)$ and the expected number of visits to State-1, i.e., $m_{11}$. Hence, the $E(I)$ is expressed as:

$$
E(I)=\frac{1}{p} \times m_{11} .
$$

Under the proposed plan the probability of acceptance of a batch in the operating characteristic curve is expressed as:

$$
L(p)=p_{12}=\frac{P\left\{Y_{i} \geq U\right\}}{1-P\left\{L<Y_{i}<U\right\}},
$$

where $L(p)$ presents the probability of acceptance of 
the batch when the probability of failure of an item is $p$. Each sampling plan must satisfy Type- 1 and Type- 2 errors. The Type- 1 error means the rejection probability of a batch when the lifetime of all the items in the batch is acceptable while the Type- 2 error means the acceptance probability of a batch when the lifetime of all the items in the batch is rejected.

At this stage, we consider different values of $L$ and $U$ to select their feasible values that satisfy Type1 and Type- 2 errors and from these feasible values, we can choose the least values for the expected number of items inspected which is calculated using Eq. (16) for a given value of $\lambda$. It is important to note that the mean ratio $\mu / \mu_{0}$ works as a quality parameter in lifetime experiments. For $\mu / \mu_{0}=1$, the acceptance probability of the lot should be greater than the producer confidence level $1-\alpha$ and smaller than the consumer risk $\beta$. So, first, we have to find out the feasible values of $L$ and $U$ to satisfy these two conditions expressed in Eqs. (17) and (18). Substituting Eq. (13) in Eq. (14) we get the following objective function $E(I)$. The average number of inspected items $E(I)$ is minimized using feasible values of $L$ and $U$.

$$
E(I)=\frac{1-\exp \left(-\left(\frac{1}{\gamma}\right)^{k}\right)}{1-\exp \left(-\left(\frac{1-\exp \left(-F\left(\frac{t}{\lambda}\right)\right)}{\gamma}\right)^{k}\right)} m_{11}
$$

subjected to the following conditions:

$$
L\left(p_{1}\right)=\frac{P\left\{Y_{i} \geq U\right\}}{1-P\left\{L<Y_{i}<U\right\}} \geq(1-\alpha),
$$

$$
L\left(p_{2}\right)=\frac{P\left\{Y_{i} \geq U\right\}}{1-P\left\{L<Y_{i}<U\right\}} \leq \beta .
$$

The values of $p_{1}$ and $p_{2}$ can be obtained using Eqs. (7) and (8) relative to producer and consumer risk respectively.

\subsection{WE distribution}

The WE distribution, being more flexible than the exponential distribution, is a key life testing model and is extensively used in engineering. We use WE distribution to check the quality of electro-mechanical products using an acceptance sampling plan. The probability density function (pdf), cumulative density function (cdf), mean $(\mu)$, probability of an item failure before the time of experiment $\left(p_{1}\right)$ and probability of non-conforming items when it fails in a test batch $\left(p_{2}\right)$ of WE distribution are presented in Table 1. The plans for different parameter values are shown in Tables 2-4.

\subsection{WL distribution}

The WL distribution is equally useful in life testing modeling and has wider applications in areas such as engineering, lifetime data, hydrology, economics, (income inequality), and others. We use WL distribution to check again the quality of electro-mechanical products using an acceptance sampling plan. The probability density function ( $p d f$ ), cumulative density function (cdf), mean $(\mu)$, probability of an item failure before the time of experiment $\left(p_{1}\right)$ and probability of non-conforming items when it fails in a test batch $\left(p_{2}\right)$

Table 1. Properties of Weibull exponential and Weibull Lomax distribution.

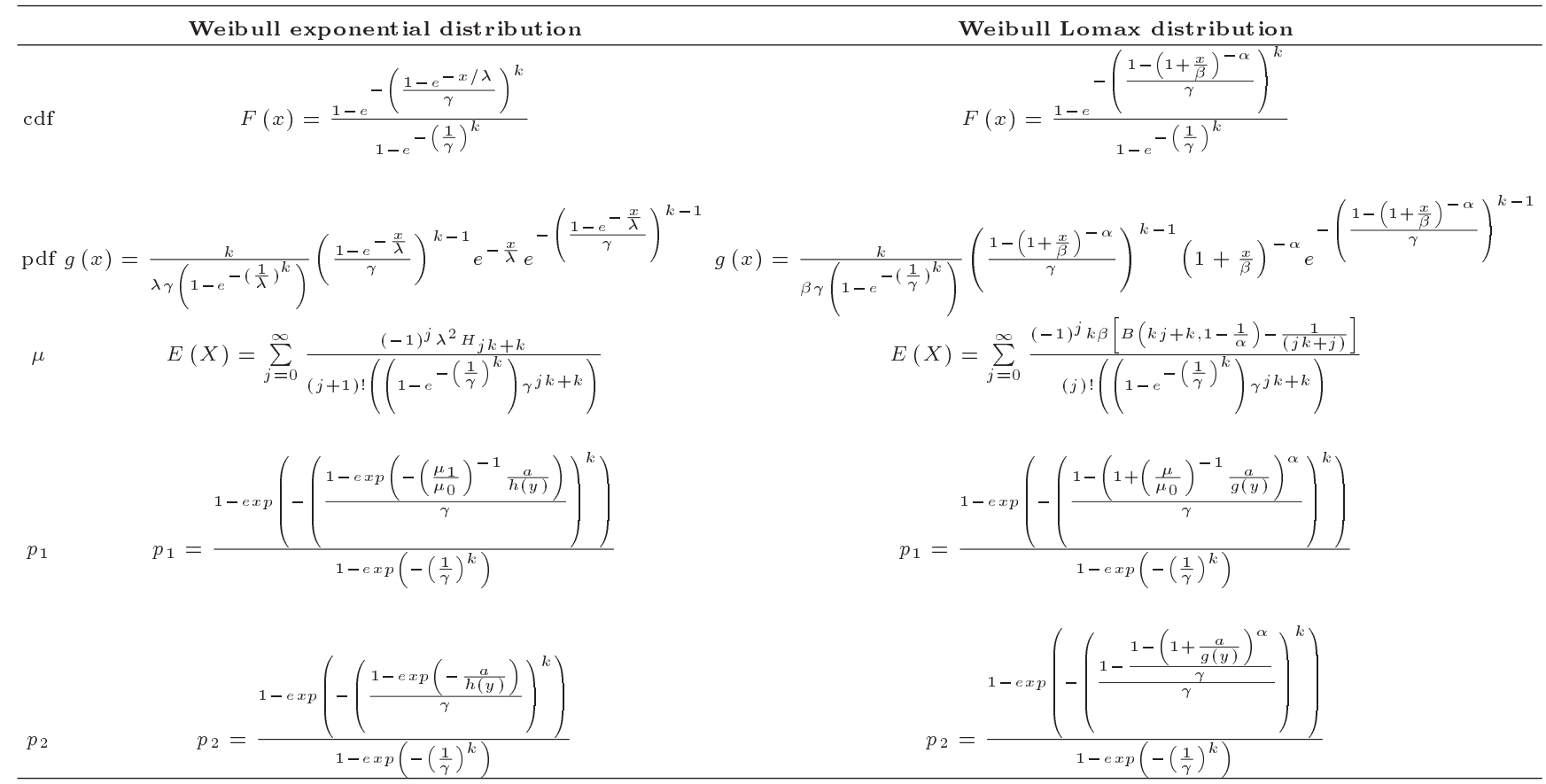


Table 2. Plan parameter values when $\gamma=0.6$ and $k=2$ for Weibull exponential distribution.

\begin{tabular}{|c|c|c|c|c|c|c|c|c|c|c|c|}
\hline \multirow[b]{2}{*}{$\boldsymbol{\beta}$} & \multirow[b]{2}{*}{$\mu / \mu_{0}$} & \multicolumn{5}{|c|}{$a=0.5$} & \multicolumn{5}{|c|}{$a=0.3$} \\
\hline & & $L$ & $U$ & $E(I)$ & $L\left(p_{1}\right)$ & $L\left(p_{2}\right)$ & $L$ & $U$ & $E(I)$ & $L\left(p_{1}\right)$ & $L\left(p_{2}\right)$ \\
\hline \multirow[t]{4}{*}{0.25} & 4 & 6 & 10 & 8.05 & 0.9555 & 0.2469 & 20 & 22 & 4.45 & 0.9519 & 0.0036 \\
\hline & 6 & 16 & 18 & 7.11 & 0.9521 & 0.0639 & 47 & 49 & 4.44 & 0.9508 & 0.0000 \\
\hline & 8 & 30 & 32 & 7.04 & 0.9505 & 0.0074 & 48 & 50 & 4.44 & 0.9713 & 0.0000 \\
\hline & 10 & 47 & 49 & 7.03 & 0.9508 & 0.0005 & 48 & 50 & 4.44 & 0.9815 & 0.0000 \\
\hline \multirow[t]{4}{*}{0.01} & 4 & 5 & 34 & 11.60 & 0.9554 & 0.0089 & 20 & 22 & 4.45 & 0.9519 & 0.0036 \\
\hline & 6 & 16 & 31 & 7.53 & 0.9503 & 0.0092 & 47 & 49 & 4.44 & 0.9508 & 0.0000 \\
\hline & 8 & 30 & 32 & 7.04 & 0.9506 & 0.0074 & 48 & 50 & 4.44 & 0.9713 & 0.0000 \\
\hline & 10 & 47 & 49 & 7.03 & 0.9508 & 0.0005 & 48 & 50 & 4.44 & 0.9815 & 0.0000 \\
\hline \multirow[t]{4}{*}{0.05} & 4 & 6 & 22 & 10.16 & 0.9521 & 0.0494 & 20 & 22 & 4.45 & 0.9519 & 0.0036 \\
\hline & 6 & 16 & 20 & 7.23 & 0.9518 & 0.0478 & 47 & 49 & 4.44 & 0.9508 & 0.0000 \\
\hline & 8 & 30 & 32 & 7.04 & 0.9506 & 0.0074 & 48 & 50 & 4.44 & 0.9713 & 0.0000 \\
\hline & 10 & 47 & 49 & 7.03 & 0.9508 & 0.0005 & 48 & 50 & 4.44 & 0.9815 & 0.0000 \\
\hline \multirow[t]{4}{*}{0.10} & 4 & 6 & 18 & 9.75 & 0.9533 & 0.0877 & 20 & 22 & 4.45 & 0.9519 & 0.0036 \\
\hline & 6 & 16 & 18 & 7.11 & 0.9521 & 0.0639 & 47 & 49 & 4.44 & 0.9508 & 0.0000 \\
\hline & 8 & 30 & 32 & 7.04 & 0.9506 & 0.0074 & 48 & 50 & 4.44 & 0.9713 & 0.0000 \\
\hline & 10 & 47 & 49 & 7.03 & 0.9508 & 0.0005 & 48 & 50 & 4.44 & 0.9815 & 0.0000 \\
\hline
\end{tabular}

Table 3. Plan parameter values when $\gamma=0.5$ and $k=2$ for Weibull exponential distribution.

\begin{tabular}{|c|c|c|c|c|c|c|c|c|c|c|c|}
\hline \multirow[b]{2}{*}{$\boldsymbol{\beta}$} & \multirow[b]{2}{*}{$\mu / \mu_{0}$} & \multicolumn{5}{|c|}{$a=0.5$} & \multicolumn{5}{|c|}{$a=0.3$} \\
\hline & & $L$ & $U$ & $E(I)$ & $L\left(p_{1}\right)$ & $L\left(p_{2}\right)$ & $L$ & $U$ & $E(I)$ & $L\left(p_{1}\right)$ & $L\left(p_{2}\right)$ \\
\hline \multirow[t]{4}{*}{0.25} & 2 & 0 & 5 & 6.13 & 0.9612 & 0.2487 & 3 & 5 & 2.22 & 0.9506 & 0.0441 \\
\hline & 4 & 4 & 6 & 2.98 & 0.9554 & 0.0785 & 16 & 17 & 2.14 & 0.9525 & 0.0000 \\
\hline & 6 & 11 & 13 & 2.86 & 0.9519 & 0.0036 & 36 & 37 & 2.14 & 0.9505 & 0.0000 \\
\hline & 8 & 21 & 23 & 2.85 & 0.9502 & 0.0000 & 48 & 50 & 2.14 & 0.9607 & 0.0000 \\
\hline \multirow[t]{4}{*}{0.01} & 2 & 0 & 8 & 7.48 & 0.9572 & 0.0833 & 2 & 8 & 2.50 & 0.9608 & 0.0074 \\
\hline & 4 & 4 & 6 & 2.98 & 0.9554 & 0.0785 & 16 & 17 & 2.14 & 0.9525 & 0.0000 \\
\hline & 6 & 11 & 13 & 2.86 & 0.9519 & 0.0036 & 36 & 37 & 2.14 & 0.9505 & 0.0000 \\
\hline & 8 & 21 & 23 & 2.85 & 0.9502 & 0.0000 & 49 & 50 & 2.14 & 0.9615 & 0.0000 \\
\hline \multirow[t]{8}{*}{0.05} & 2 & 0 & 10 & 7.86 & 0.9541 & 0.0369 & 3 & 5 & 2.22 & 0.9508 & 0.0441 \\
\hline & 4 & 4 & 8 & 3.12 & 0.9546 & 0.0347 & 16 & 17 & 2.14 & 0.9525 & 0.0000 \\
\hline & 6 & 11 & 13 & 2.86 & 0.9519 & 0.0036 & 36 & 37 & 2.14 & 0.9505 & 0.0000 \\
\hline & 8 & 21 & 23 & 2.86 & 0.9502 & 0.0000 & 48 & 50 & 2.14 & 0.9607 & 0.0000 \\
\hline & 2 & - & - & - & - & - & 3 & 5 & 2.22 & 0.9506 & 0.0441 \\
\hline & 4 & 4 & 11 & 3.20 & 0.9534 & 0.0097 & 16 & 17 & 2.14 & 0.9525 & 0.0000 \\
\hline & 6 & 11 & 13 & 2.86 & 0.9519 & 0.0036 & 36 & 37 & 2.14 & 0.9505 & 0.0000 \\
\hline & 8 & 23 & 24 & 2.86 & 0.9501 & 0.0000 & 48 & 50 & 2.14 & 0.9607 & 0.0000 \\
\hline
\end{tabular}

of WL distribution are given in Table 1. In Table 1:

$$
\begin{aligned}
& h(y)=\int_{0}^{1} \frac{k \ln (1-\gamma y) y^{k-1} e^{-y^{k}}}{\gamma\left(1-e^{-\left(\frac{1}{\gamma}\right)^{k}}\right)} d y, \\
& g(y)= \\
& \int_{0}^{1} \frac{k \alpha(y-1) y^{-(\alpha+1)}\left(\frac{1-y^{-\alpha}}{\gamma}\right)^{k-1} e^{-\left(\frac{1-y^{-\alpha}}{\gamma}\right)^{k}}}{\gamma\left(1-e^{-\left(\frac{1}{\gamma}\right)^{k}}\right)} d y
\end{aligned}
$$

are computed using numerical integration whereas $H_{j k+k}$ represents the $(j k-k)$ th harmonic number defined as $H_{j k-k}=\sum_{i=1}^{j k-k} \frac{1}{i}$. The plans for different parameter values are shown in Tables $5-7$. The plan parameters of the two distributions can be calculated for different values of termination time ratio $(a=0.5,0.3)$, mean ratio $\left(\frac{\mu}{\mu_{0}}=4,6,8,10\right)$, and consumer risk $(\beta=$ $0.25,0.01,0.05,0.10)$ of both WE and WL distributions. We take the values of the shape parameter $(k)$ 
Table 4. Plan parameter values when $\gamma=0.5$ and $k=4$ for Weibull exponential distribution.

\begin{tabular}{|c|c|c|c|c|c|c|c|c|c|c|c|}
\hline \multirow[b]{2}{*}{$\boldsymbol{\beta}$} & \multirow[b]{2}{*}{$\mu / \mu_{0}$} & \multicolumn{5}{|c|}{$a=0.5$} & \multicolumn{5}{|c|}{$a=0.3$} \\
\hline & & $L$ & $U$ & $E(I)$ & $L\left(p_{1}\right)$ & $L\left(p_{2}\right)$ & $L$ & $U$ & $E(I)$ & $L\left(p_{1}\right)$ & $L\left(p_{2}\right)$ \\
\hline \multirow[t]{4}{*}{0.25} & 2 & 19 & 21 & 9.73 & 0.9501 & 0.1008 & 48 & 50 & 3.98 & 0.9823 & 0.0000 \\
\hline & 4 & 48 & 50 & 9.62 & 0.9912 & 0.0041 & 48 & 50 & 3.98 & 0.9988 & 0.0000 \\
\hline & 6 & 48 & 50 & 9.62 & 0.9981 & 0.0041 & 48 & 50 & 3.98 & 0.9997 & 0.0000 \\
\hline & 8 & 48 & 50 & 9.62 & 0.9994 & 0.0041 & 48 & 50 & 3.98 & 0.9997 & 0.0000 \\
\hline \multirow[t]{4}{*}{0.01} & 2 & 17 & 44 & 11.1 & 0.9521 & 0.0092 & 48 & 50 & 3.98 & 0.9823 & 0.0000 \\
\hline & 4 & 48 & 50 & 9.62 & 0.9912 & 0.0041 & 48 & 50 & 3.98 & 0.9988 & 0.0000 \\
\hline & 6 & 48 & 50 & 9.62 & 0.9981 & 0.0041 & 48 & 50 & 3.98 & 0.9997 & 0.0000 \\
\hline & 8 & 48 & 50 & 9.62 & 0.9994 & 0.0041 & 48 & 50 & 3.98 & 0.9997 & 0.0000 \\
\hline \multirow[t]{4}{*}{0.05} & 2 & 18 & 29 & 10.5 & 0.9514 & 0.0451 & 48 & 50 & 3.98 & 0.9823 & 0.0000 \\
\hline & 4 & 48 & 50 & 9.62 & 0.9912 & 0.0041 & 48 & 50 & 3.98 & 0.9988 & 0.0000 \\
\hline & 6 & 48 & 50 & 9.62 & 0.9981 & 0.0041 & 48 & 50 & 3.98 & 0.9997 & 0.0000 \\
\hline & 8 & 48 & 50 & 9.62 & 0.9994 & 0.0041 & 48 & 50 & 3.98 & 0.9997 & 0.0000 \\
\hline \multirow[t]{4}{*}{0.10} & 2 & 18 & 22 & 9.96 & 0.9522 & 0.0925 & 48 & 50 & 3.98 & 0.9823 & 0.0000 \\
\hline & 4 & 48 & 50 & 9.62 & 0.9912 & 0.0041 & 48 & 50 & 3.98 & 0.9988 & 0.0000 \\
\hline & 6 & 48 & 50 & 9.62 & 0.9981 & 0.0041 & 48 & 50 & 3.98 & 0.9997 & 0.0000 \\
\hline & 8 & 48 & 50 & 9.62 & 0.9994 & 0.0041 & 48 & 50 & 3.98 & 0.9997 & 0.0000 \\
\hline
\end{tabular}

Table 5. Plan parameter values when $\gamma=2, \alpha=1.5$, and $k=3$ for Weibull Lomax distribution.

\begin{tabular}{|c|c|c|c|c|c|c|c|c|c|c|c|}
\hline \multirow[b]{2}{*}{$\boldsymbol{\beta}$} & \multirow[b]{2}{*}{$\mu / \mu_{0}$} & \multicolumn{5}{|c|}{$a=0.5$} & \multicolumn{5}{|c|}{$a=0.3$} \\
\hline & & $L$ & $U$ & $E(I)$ & $L\left(p_{1}\right)$ & $L\left(p_{2}\right)$ & $L$ & $U$ & $E(I)$ & $L\left(p_{1}\right)$ & $L\left(p_{2}\right)$ \\
\hline \multirow[t]{4}{*}{0.25} & 2 & 5 & 7 & 3.34 & 0.9537 & 0.0771 & 25 & 26 & 2.08 & 0.9512 & 0.0000 \\
\hline & 4 & 39 & 41 & 3.23 & 0.9505 & 0.0000 & 48 & 50 & 2.08 & 0.9851 & 0.0000 \\
\hline & 6 & 48 & 50 & 3.23 & 0.9800 & 0.0000 & 48 & 50 & 2.08 & 0.9953 & 0.0000 \\
\hline & 8 & 48 & 50 & 3.23 & 0.9911 & 0.0000 & 48 & 50 & 2.08 & 0.9979 & 0.0000 \\
\hline \multirow[t]{4}{*}{0.01} & 2 & 5 & 13 & 3.59 & 0.9515 & 0.0089 & 25 & 26 & 2.08 & 0.9512 & 0.0000 \\
\hline & 4 & 39 & 41 & 3.23 & 0.9505 & 0.0000 & 25 & 26 & 2.08 & 0.9512 & 0.0000 \\
\hline & 6 & 48 & 50 & 3.23 & 0.9800 & 0.0000 & 48 & 50 & 2.08 & 0.9851 & 0.0000 \\
\hline & 8 & 48 & 50 & 3.23 & 0.9911 & 0.0000 & 48 & 50 & 2.08 & 0.9953 & 0.0000 \\
\hline \multirow[t]{4}{*}{0.05} & 2 & 5 & 9 & 3.48 & 0.9530 & 0.0383 & 48 & 50 & 2.08 & 0.9979 & 0.0000 \\
\hline & 4 & 41 & 42 & 3.23 & 0.9505 & 0.0000 & 25 & 26 & 2.08 & 0.9512 & 0.0000 \\
\hline & 6 & 48 & 50 & 3.23 & 0.9800 & 0.0000 & 48 & 50 & 2.08 & 0.9851 & 0.0000 \\
\hline & 8 & 48 & 50 & 3.23 & 0.9911 & 0.0000 & 48 & 50 & 2.08 & 0.9953 & 0.0000 \\
\hline \multirow[t]{4}{*}{0.10} & 2 & 5 & 7 & 3.34 & 0.9537 & 0.0771 & 48 & 50 & 2.08 & 0.9979 & 0.0000 \\
\hline & 4 & 39 & 41 & 3.23 & 0.9505 & 0.0000 & 25 & 26 & 2.08 & 0.9512 & 0.0000 \\
\hline & 6 & 48 & 50 & 3.23 & 0.9800 & 0.0000 & 48 & 50 & 2.08 & 0.9851 & 0.0000 \\
\hline & 8 & 48 & 50 & 3.23 & 0.9911 & 0.0000 & 48 & 50 & 2.08 & 0.9953 & 0.0000 \\
\hline
\end{tabular}

as 2 and 4 while the values of the scale parameter $(\gamma)$ as 0.6 and 0.5 in the WE distribution as shown in Tables 2, 3, and 4, respectively. We couldn't find plan parameters for the shape parameter values 2 and 4 in some cases during simulation. It is worth mentioning that the plan parameters are not also found when the shape parameter is 1 . We take the values of the shape parameters $(k)$ and $(\alpha)$ as 3,2 and $1.5,3,2$, respectively whereas the values of the scale parameter $(\gamma)$ as 3 and 2 in the case of the WL distribution as shown in Tables 5,6 , and 7 , respectively.
The empty cells show that the plan parameters don't satisfy the conditions. The findings of the above tables for WE and WL distributions can be stated as:

1. $E(I)$ decreases when the termination time ratio increases;

2. $E(I)$ decreases when the mean ratio $\mu / \mu_{0}$ increases;

3. $E(I)$ decreases as $a$ (a constant) decreases;

4. $E(I)$ increases as the value of the shape parameter increases. 
Table 6. Plan parameter values when $\gamma=2, \alpha=3$, and $k=2$ for Weibull Lomax distribution.

\begin{tabular}{|c|c|c|c|c|c|c|c|c|c|c|c|}
\hline \multirow[b]{2}{*}{$\beta$} & \multirow[b]{2}{*}{$\mu / \mu_{0}$} & \multicolumn{5}{|c|}{$a=0.5$} & \multicolumn{5}{|c|}{$a=0.3$} \\
\hline & & $\boldsymbol{L}$ & $U$ & $E(I)$ & $L\left(p_{1}\right)$ & $L\left(p_{2}\right)$ & $\boldsymbol{L}$ & $U$ & $E(I)$ & $L\left(p_{1}\right)$ & $L\left(p_{2}\right)$ \\
\hline \multirow[t]{4}{*}{0.25} & 2 & 0 & 3 & 2.83 & 0.9716 & 0.1405 & 5 & 6 & 1.44 & 0.9573 & 0.0007 \\
\hline & 4 & 7 & 8 & 1.82 & 0.9550 & 0.0016 & 19 & 20 & 1.43 & 0.9500 & 0.0000 \\
\hline & 6 & 15 & 16 & 1.82 & 0.9522 & 0.0000 & 38 & 40 & 1.43 & 0.9510 & 0.0000 \\
\hline & 8 & 26 & 27 & 1.82 & 0.9513 & 0.0000 & 34 & 48 & 1.43 & 0.9744 & 0.0000 \\
\hline \multirow[t]{4}{*}{0.01} & 2 & 0 & 7 & 3.26 & 0.9685 & 0.0065 & 5 & 6 & 1.44 & 0.9573 & 0.0007 \\
\hline & 4 & 7 & 8 & 1.82 & 0.9550 & 0.0016 & 19 & 20 & 1.43 & 0.9500 & 0.0000 \\
\hline & 6 & 15 & 16 & 1.82 & 0.9522 & 0.0000 & 34 & 44 & 1.43 & 0.9555 & 0.0000 \\
\hline & 8 & 26 & 27 & 1.82 & 0.9513 & 0.0000 & 40 & 47 & 1.43 & 0.9703 & 0.0000 \\
\hline \multirow[t]{4}{*}{0.05} & 2 & 0 & 5 & 3.18 & 0.9701 & 0.0318 & 5 & 6 & 1.44 & 0.9573 & 0.0007 \\
\hline & 4 & 7 & 8 & 1.82 & 0.9550 & 0.0016 & 19 & 20 & 1.43 & 0.9500 & 0.0000 \\
\hline & 6 & 15 & 16 & 1.82 & 0.9522 & 0.0000 & 32 & 33 & 1.43 & 0.9608 & 0.0000 \\
\hline & 8 & 26 & 27 & 1.82 & 0.9513 & 0.0000 & 34 & 44 & 1.43 & 0.9745 & 0.0000 \\
\hline \multirow[t]{4}{*}{0.10} & 2 & 0 & 4 & 3.06 & 0.9709 & 0.0683 & 5 & 6 & 1.43 & 0.9573 & 0.0007 \\
\hline & 4 & 7 & 8 & 1.82 & 0.9550 & 0.0016 & 19 & 20 & 1.43 & 0.9500 & 0.0000 \\
\hline & 6 & 15 & 16 & 1.82 & 0.9522 & 0.0000 & 37 & 50 & 1.43 & 0.9516 & 0.0000 \\
\hline & 8 & 26 & 27 & 1.82 & 0.9513 & 0.0000 & 37 & 42 & 1.43 & 0.9725 & 0.0000 \\
\hline
\end{tabular}

Table 7. Plan parameter values when $\gamma=3, \alpha=2$, and $k=2$ for Weibull Lomax distribution.

\begin{tabular}{|c|c|c|c|c|c|c|c|c|c|c|c|}
\hline \multirow[b]{2}{*}{$\beta$} & \multirow[b]{2}{*}{$\mu / \mu_{0}$} & \multicolumn{5}{|c|}{$a=0.5$} & \multicolumn{5}{|c|}{$a=0.3$} \\
\hline & & $L$ & $U$ & $E(I)$ & $L\left(p_{1}\right)$ & $L\left(p_{2}\right)$ & $\boldsymbol{L}$ & $U$ & $E(I)$ & $L\left(p_{1}\right)$ & $L\left(p_{2}\right)$ \\
\hline \multirow[t]{4}{*}{0.25} & 2 & 2 & 3 & 2.23 & 0.9723 & 0.1181 & 4 & 6 & 1.47 & 0.9505 & 0.0010 \\
\hline & 4 & 8 & 9 & 1.86 & 0.9504 & 0.0009 & 19 & 20 & 1.46 & 0.9523 & 0.0000 \\
\hline & 6 & 16 & 17 & 1.85 & 0.9513 & 0.0000 & 40 & 44 & 1.46 & 0.9506 & 0.0000 \\
\hline & 8 & 27 & 28 & 1.85 & 0.9516 & 0.0000 & 39 & 48 & 1.46 & 0.9722 & 0.0000 \\
\hline \multirow[t]{4}{*}{0.01} & 2 & 0 & 7 & 3.42 & 0.9707 & 0.0082 & 4 & 6 & 1.46 & 0.9505 & 0.0010 \\
\hline & 4 & 8 & 9 & 1.86 & 0.9504 & 0.0009 & 19 & 20 & 1.46 & 0.9523 & 0.0000 \\
\hline & 6 & 16 & 17 & 1.85 & 0.9513 & 0.0000 & 36 & 50 & 1.46 & 0.9548 & 0.0000 \\
\hline & 8 & 27 & 28 & 1.85 & 0.9516 & 0.0000 & 36 & 49 & 1.46 & 0.9742 & 0.0000 \\
\hline \multirow[t]{4}{*}{0.05} & 2 & 0 & 5 & 3.31 & 0.9722 & 0.0374 & 4 & 6 & 1.46 & 0.9505 & 0.0010 \\
\hline & 4 & 8 & 9 & 1.86 & 0.9504 & 0.0001 & 19 & 20 & 1.46 & 0.9523 & 0.0000 \\
\hline & 6 & 16 & 17 & 1.85 & 0.9513 & 0.0000 & 36 & 50 & 1.46 & 0.9548 & 0.0000 \\
\hline & 8 & 27 & 28 & 1.85 & 0.9516 & 0.0000 & 36 & 49 & 1.46 & 0.9742 & 0.0000 \\
\hline \multirow[t]{4}{*}{0.10} & 2 & 0 & 4 & 3.183 & 0.9728 & 0.0776 & 4 & 7 & 1.46 & 0.9505 & 0.0010 \\
\hline & 4 & 8 & 9 & 1.86 & 0.9504 & 0.0009 & 4 & 6 & 1.46 & 0.9505 & 0.0010 \\
\hline & 6 & 16 & 17 & 1.85 & 0.9513 & 0.0000 & 19 & 20 & 1.46 & 0.9523 & 0.0000 \\
\hline & 8 & 27 & 28 & 1.85 & 0.9516 & 0.0000 & 34 & 37 & 1.46 & 0.9577 & 0.0000 \\
\hline
\end{tabular}




\section{Plans assessment}

The shape parameter of the failure rate should be closely monitored while applying the proposed acceptance sampling plan. If it is increasing then the sampling plan is reasonable. As the frequency of the inspected items increases, the quality of conforming items improves. A comparison between the proposed plans and an already existing plan proposed by Aslam et al. [11] in terms of $E(I)$ for $a=0.5$ and $k=2$ is presented in Table 8. A plan with lower values of $E(I)$ is considered to be more efficient. It is evident from Table 8 that the proposed plan has lower values of $E(I)$ for both WE and WL distributions than those of the existing plan. For instance, for the mean ratio $\mu / \mu_{0}=4$ and the consumer risk $\beta=0.25$, the values of $E(I)$ for WE and WL distributions are 6.13 and 2.23 respectively while the value of $E(I)$ for the acceptance plan proposed by Aslam et al. [11] is 9.49. Hence, the proposed plan is performing better than the existing one.

\section{Application in electronic industry}

The data, taken from Juran and Gryna [37], shows the failure times (hours) of 107 units of a piece of electronic equipment. Schneider et al. [38] suggested that there is an approximately 20 hours early failure period and about a 100-hour wear-out period i.e., the period between these hours being shown fairly constant. They considered the expected number of failures at the end of the early period and at the beginning of the wear-out period. In this example, the parameters are estimated by the Maximum Likelihood Estimation (MLE) method for WE, WL, and Weibull distributions using R-code and are given in Table 9 .

The MLEs from Table 9 are used to find the expected number of conforming items, e.g. for $\mu / \mu_{0}=$ $4, a=0.5,1-\alpha=0.95, \beta=0.25$, we get $L=16$, $U=25$, and $E(I)=1.0$ for the WL distribution which is the smallest value of $E(I)$ as compared to those of WE and Weibull distributions.

The practical implementation of the proposed

Table 8. Comparison of the proposed plan with the existing one in terms of $E(I)$ for $a=0.5$ and $k=2$.

\begin{tabular}{|c|c|c|c|c|}
\hline $\boldsymbol{\beta}$ & $\mu / \mu_{0}$ & $\begin{array}{c}\text { Proposed plan } \\
\text { based on WE } \\
E(I)\end{array}$ & $\begin{array}{c}\text { Proposed plan } \\
\text { based on WL } \\
E(I)\end{array}$ & $\begin{array}{c}\text { Existing plan } \\
\text { by Aslam et al. [11] } \\
E(I)\end{array}$ \\
\hline \multirow{4}{*}{0.25} & 4 & 6.13 & 2.23 & 9.49 \\
\hline & 6 & 2.98 & 1.86 & 6.47 \\
\hline & 8 & 2.86 & 1.85 & 6.33 \\
\hline & 10 & 2.85 & 1.85 & 6.29 \\
\hline \multirow{4}{*}{0.10} & 4 & 7.48 & 3.42 & 11.37 \\
\hline & 6 & 2.98 & 1.86 & 6.89 \\
\hline & 8 & 2.86 & 1.85 & 6.33 \\
\hline & 10 & 2.85 & 1.85 & 6.29 \\
\hline \multirow{4}{*}{0.05} & 4 & 7.86 & 3.31 & 14.82 \\
\hline & 6 & 3.12 & 1.86 & 7.31 \\
\hline & 8 & 2.86 & 1.85 & 6.33 \\
\hline & 10 & 2.86 & 1.85 & 6.29 \\
\hline \multirow{4}{*}{0.01} & 4 & - & 3.18 & 15.36 \\
\hline & 6 & 3.20 & 1.86 & 7.88 \\
\hline & 8 & 2.86 & 1.85 & 6.51 \\
\hline & 10 & 2.86 & 1.85 & 6.29 \\
\hline
\end{tabular}

Table 9. MLEs of Weibull exponential, Weibull Lomax, and Weibull distributions for failure times (hours) of 107 units of a piece of electronic equipment along with negative log-likelihood values.

\begin{tabular}{lcccccc}
\hline \multirow{2}{*}{ Model } & \multicolumn{5}{c}{ Estimates } & Negative log \\
\cline { 2 - 6 } & $\boldsymbol{\alpha}$ & $\boldsymbol{\beta}$ & $\boldsymbol{k}$ & $\boldsymbol{\gamma}$ & $\boldsymbol{\lambda}$ & likelihood values \\
\hline Weibull exponential & - & - & 0.896 & 2.120 & 42.959 & -52.09 \\
Weibull lomax & 1.925 & 0.017 & 0.049 & 2.473 & - & -53.67 \\
Weibull & 2.012 & 25.820 & - & - & - & -53.78 \\
\hline
\end{tabular}


Table 10. Expected number of conforming items using estimated parameter values for Weibull exponential, Weibull Lomax, and Weibull distributions.

\begin{tabular}{|c|c|c|c|c|c|c|}
\hline \multirow[b]{2}{*}{$\beta$} & \multicolumn{6}{|c|}{ Weibull exponential } \\
\hline & $\mu / \mu_{0}$ & $L$ & $U$ & $E(I)$ & $L\left(p_{1}\right)$ & $L\left(p_{2}\right)$ \\
\hline \multirow[t]{2}{*}{0.25} & 4 & 0 & 1 & 1.2971 & 1.0000 & 0.0000 \\
\hline & 6 & 0 & 1 & 1.2971 & 1.0000 & 0.0000 \\
\hline \multirow[t]{3}{*}{0.05} & 4 & 0 & 1 & 1.2971 & 1.0000 & 0.0000 \\
\hline & 6 & 0 & 1 & 1.2971 & 1.0000 & 0.0000 \\
\hline & \multicolumn{6}{|c|}{ Weibull Lomax } \\
\hline \multirow[t]{2}{*}{0.25} & 4 & 16 & 25 & 1.0000 & 1.0000 & 0.0000 \\
\hline & 6 & 4 & 11 & 1.0000 & 1.0000 & 0.0000 \\
\hline \multirow[t]{3}{*}{0.05} & 4 & 15 & 47 & 1.0000 & 1.0000 & 0.0000 \\
\hline & 6 & 6 & 16 & 1.0000 & 1.0000 & 0.0000 \\
\hline & \multicolumn{6}{|c|}{ Weibull } \\
\hline \multirow[t]{2}{*}{0.25} & 4 & 2 & 20 & 12.07 & 0.9502 & 0.0423 \\
\hline & 6 & 8 & 16 & 6.43 & 0.9503 & 0.0495 \\
\hline \multirow[t]{2}{*}{0.05} & 4 & 2 & 12 & 9.58 & 0.9607 & 0.2397 \\
\hline & 6 & 8 & 10 & 5.79 & 0.9518 & 0.1477 \\
\hline
\end{tabular}

plan considering the WL distribution for the inspection of electronic equipment is illustrated as follows. Let $Y_{i}$ be the number of conforming items between the successive second and third non-conforming items. The states involved in this process are defined under the time truncated life tests as follows:

State-1: $Y_{i}$ is between the two control thresholds (i.e., $16<Y_{i}<25$ ); thus testing of the lifetime of items continues;

State-2: $Y_{i}$ is greater than or equal to the upper control threshold (i.e., $Y_{i} \geq 25$ ); hence the batch is accepted;

State-3: $Y_{i}$ is less than or equal to the lower control threshold (i.e., $Y_{i} \leq 16$ ); hence the batch is rejected.

From Table 10, it is obvious that the proposed plan has lower values of $E(I)$ for both WE and WL distributions as compared to the Weibull distribution showing that the Weibull-X family performs better. Moreover, the larger negative log-likelihood value for the proposed Weibull-X family than for the Weibull distribution confirms the better model performance.

\section{Conclusion}

The acceptance sampling plan is, indeed, the core qualitative element in the industrial as well as service sector since it particularly focuses on the reduction in manufacturing tolerances and the addition of specifications. Several sampling plans have been proposed using standard distributions in the literature. In this research, sampling plans based on the Weibull$\mathrm{X}$ family of distributions are proposed under the time truncated life test. The use of the Weibull-X family of distributions not only distinguishes the present work but it paves the way for extending it to any other family of distributions. Moreover, the distribution parameters are estimated using the maximum likelihood method contrary to the existing plans where authors select arbitrary values of the parameters. In the real-life example, the parameters obtained by the maximum likelihood method for Weibull Exponential and Weibull Lomax distributions are used to design the acceptance sampling plans and calculate the expected number of inspected items $E(I)$ The smaller values of $E(I)$ of the proposed plans than those of the sampling plan proposed by Aslam et al. [11] suggest a smaller length of the transient state. This will lead to a prompt decision of accepting or rejecting a batch even after inspecting a smaller number of items. As the sample size becomes larger, the time and the cost of inspection of the product also become larger. So, it is recommended to use the proposed plan in the industry to save inspection costs and effort while protecting the producer and consumer risks.

The proposed plan can be applied in engineering especially in failure time modeling and reliability analysis to check the quality of electro-mechanical products whose lifespan follows the Weibull Exponential and Weibull Lomax distributions.

\section{Acknowledgments}

The authors are thankful to the editorial board and the two referees for their valuable comments and suggestions which certainly helped to improve the paper.

\section{Notations}

$p \quad$ Probability of nonconforming items when it fails in a test batch

$\alpha \quad$ Probability of Type-I error in decision making

$\beta \quad$ Probability of Type-II error in decision making

$p_{i j} \quad$ Probability of moving from state $i$ to state $j$ in a single-stage

$p_{1} \quad$ Probability of conforming items when it fails in a test batch

$p_{2} \quad$ Probability of nonconforming items when it fails in a test batch

$A \quad$ Identity matrix depicting the probabilities of staying in a state 
O Null matrix depicting the probabilities of not reaching an absorbing state

$Q \quad$ Square matrix comprising transition probabilities of going from a non-absorbing state to another non-absorbing state

$R \quad$ Matrix comprising probabilities of moving from a non-absorbing state to an absorbing state

Fundamental matrix comprising expected transitions from a nonabsorbing state to another nonabsorbing state before the occurrence of absorption

$F \quad$ Absorption probability matrix comprising long-run probabilities of transitions from non-absorbing states to absorbing states

$U \quad$ Upper threshold number of defective items

$L \quad$ Lower threshold number of defective items

$I \quad$ Number of items inspected

a Termination time ratio

$t_{0} \quad$ Total testing time for an item in an experiment

$\mu_{0}$

$\mu_{1}$

Mean lifetime of failure of a nonconforming item

Mean lifetime of failure of a conforming item

\section{References}

1. Balakrishnan, N., Leiva, V., and Lopez, J. "Acceptance sampling plans from truncated life tests based on the generalized Birnbaum-Saunders distribution", Communications in Statistics-Simulation and Computation, 36(3), pp. 645-656 (2007).

2. Bourke, P.D. "A continuous sampling plan using CUSUMs", Journal of Applied Statistics, 29(8), pp. 1121-1133 (2002).

3. Bourke, P.D. "A continuous sampling plan using sums of conforming run lengths", Quality and Reliability Engineering International, 19(1), pp. 53-66 (2003).

4. Niaki, S.A. and Fallahnezhad, M.S. "Designing an optimum acceptance sampling plan using Bayesian inferences and a stochastic dynamic programming approach", Scientia Iranica, Transaction E, Industrial Engineering, 16(1), pp. 19-25 (2009).

5. Mirabi, M. and Fallahnezhad, M.S. "Analysing acceptance sampling plans by Markov chains", South African Journal of Industrial Engineering, 23(1), pp. 151-161 (2012).

6. Fallahnezhad, M.S. "A new Markov chain based acceptance sampling policy via the minimum angle method",
Iranian Journal of Operations Research, 3(1), pp. 104111 (2012).

7. Fallahnezhad, M.S. and Niaki, S. "A new machine replacement policy based on number of defective items and Markov chains", Iranian Journal of Operations Research, 2(2), pp. 17-28 (2011).

8. Balamurali, S., Aslam, M., Ahmad, L., et al. "A mixed double sampling plan based on $C_{p k}$ ", Communications in Statistics-Theory and Methods, 49(8), pp. 18401857 (2020). DOI: 10.1080/03610926.2019.1565836

9. Baklizi, A. "Acceptance sampling based on truncated life tests in the Pareto distribution of the second kind", Advances and Applications in Statistics, 3(1), pp. 3348 (2003).

10. Aslam, M., Jun, C.-H., and Ahmad, M. "A group sampling plan based on truncated life test for Gamma distributed items", Pakistan Journal of Statistics, $\mathbf{2 5}$ (3), pp. 333-340 (2009).

11. Aslam, M., Nezhad, M.S.F., and Azam, M. "Decision procedure for the Weibull distribution based on run lengths of conforming items", Journal of Testing and Evaluation, 41(5), pp. 826-832 (2013).

12. Chowdhury, S. "Acceptance sampling plans based on truncated life test for the generalized Weibull model", IEEE International Conference on Industrial Engineering and Engineering Management (IEEM), pp. 886-889 (2016). DOI: 10.1109/IEEM.2016.7798004

13. Shahbaz, S.H., Khan, K., and Shahbaz, M.Q. "Acceptance sampling plans for finite and infinite lot size under power lindley distribution", Symmetry, 10, p. 496 (2018).

14. Al-Omari, A., Al-Nasser, A., and Ciavolino, E. "Acceptance sampling plans based on truncated life tests for Rama distribution", International Journal of Quality \& Reliability Management, 36(7), pp. 1181-1191 (2019).

15. Mahdy, M. and Ahmed, B. "New distributions in designing of double acceptance sampling plan with application", Pakistan Journal of Statistics and Operation Research, 14(2), pp. 333-346 (2018).

16. Fallahnezhad, M.S. and Saredorahi, F.Z. "A new policy for designing acceptance sampling plan based on Bayesian inference in the presence of inspection errors", Communications in Statistics-Theory and Methods, 47(21), pp. 5307-5318 (2018).

17. Fallahnezhad, M.S. and Seifi, S. "Repetitive group sampling plan based on the process capability index for the lot acceptance problem", Journal of Statistical Computation and Simulation, 87(1), pp. 29-41 (2017).

18. Fallahnezhad, M.S. and Yazdi, A.A. "An optimization model for economic design of sampling plans based on conforming run length considering outgoing quality", Communications in Statistics-Theory and Methods, 46(5), pp. 2202-2211 (2017).

19. Rasay, H., Pourgharibshahi, M., and Fallahnezhad, M.S. "Sequential sampling plan in the truncated life test for Weibull distribution", Journal of Testing and Evaluation, 46(2), pp. 693-703 (2018). 
20. Fallahnezhad, M.S. and Saredorahi, F.Z. "Designing an economically optimal repetitive group-sampling plan based on loss functions", Communications in Statistics-Simulation and Computation, 47(3), pp. 783-799 (2018).

21. Al-Omari, A.I. and Al-Hadhrami, S.A. "Acceptance sampling plans based on truncated life tests for extended exponential distribution", Kuwait Journal of Science, 45(2), pp. 89-99 (2018).

22. Al-Omari, A.I. "Acceptance sampling plans based on truncated life tests for Sushila distribution", Journal of Mathematical and Fundamental Sciences, 50(1), pp. 72-83 (2018).

23. Aslam, M., Jeyadurga, P., Balamurali, S., et al. "Timetruncated group plan under a Weibull distribution based on neutrosophic statistics", Mathematics, 7(10) p. 905 (2019).

24. Singh, N. and Buttar, G.S. "Sequential sampling plan based on truncated life test for generalized exponential distribution", International Journal on Emerging Technologies, 10(4), pp. 119-124 (2019).

25. Al-Nasser, A.D. and Obeidat, M. "Acceptance sampling plans from truncated life test based on Tsallis q-exponential distribution", Journal of Applied Statistics, 47(4), pp. 685-697 (2020).

26. Divecha, J. and Raykundaliya, D.P. "Three economical life test acceptance sampling plans", Communications in Statistics-Simulation and Computation, 51(6), pp. 3305-3323 (2022). DOI: 10.1080/03610918.2020.1711948

27. Luko, S.N. "A review of the Weibull distribution and selected engineering applications", Journal of Commercial Vehicles, 108(2), pp. 398-412 (1999).

28. Mandouh, R. "Lomax-modified Weibull distribution: A new generalization", Journal of Advances in Mathematics and Computer Science, 27(1), pp. 1-7, (2018). https://doi.org/10.9734/JAMCS/2018/38975

29. Hanook, S., Shahbaz, M.Q., Mohsin, M., et al. "A note on beta Inverse-Weibull distribution", Communications in Statistics-Theory and Methods, 42(2), pp. 320-335 (2013).

30. Cordeiro, G.M. and de Castro, M. "A new family of generalized distributions", Journal of Statistical Computation and Simulation, 81(7), pp. 883-898 (2011).

31. Ahsanullah, M., Shahbaz, S., Shahbaz, M.Q., et al. "Concomitants of upper record statistics for bivariate Pseudo-Weibull distribution", App. \& Applied Math, 5(10), pp. 1379-1388 (2010).

32. Alzaatreh, A., Lee, C., and Famoye, F. "A new method for generating families of continuous distributions", Metron, 71(1), pp. 63-79 (2013).

33. Aljarrah, M.A., Lee, C., and Famoye, F. "On generating TX family of distributions using quantile functions", Journal of Statistical Distributions and Applications, 1(2), pp. 1-17 (2014). https://doi.org/10.1186/2195-5832-1-2
34. Rather, N. and Rather, T. "New generalizations of exponential distribution with applications", Journal of Probability and Statistics, Article ID 2106748, pp. 1-9 (2017). http://doi.org/10.1155/2017/2106748

35. Mahdavi, A. and Oliveira Silva, G. "A method to expand family of continuous distributions based on truncated distributions", Journal of Statistical Research of Iran, 13(2), pp. 231-247 (2017).

36. Bowling, S.R., Khasawneh, M.T., Kaewkuekool, S., et al. "A Markovian approach to determining optimum process target levels for a multi-stage serial production system", European Journal of Operational Research, 159, pp. 636-650 (2004).

37. Juran, J. and Gryna, F., Quality Planing and Analysis, McGraw-Hill, New York (1980).

38. Schneider, H., Lin, B.-S., and O'Cinneide, C. "Comparison of nonparameteric estimators for the renewal function", Applied Statistics, 39(1), pp. 55-61 (1990).

\section{Biographies}

Mian Muhammad Farooq is a $\mathrm{PhD}$ scholar at the Department of Statistics, COMSATS University Islamabad (CUI), Lahore Campus. His area of research is Distribution theory, Survey Sampling, and Statistical quality control. He completed his M. Phil in Statistics from NCBA\&E in 2003 with distinction, with a thesis in Survey Sampling. Currently, he is serving CUI, Lahore campus as a lecturer.

Ayesha Bashair is an MS scholar at the Department of Statistics, COMSATS University Islamabad, Lahore Campus. She received her BS in Statistics from the Lahore College for Woman University, Lahore. Her research interests are computer simulation and Applied statistics.

Muhammad Mohsin is working as Chairman and Head of the Department of Statistics at COMSATS University Islamabad, Lahore campus. His areas of interest are Distribution theory, Mathematical statistics, Statistical modeling, Bayesian Inference, Meta-analysis, and Statistical quality control. He has done his Postdoc from École Polytechnique Fédérale de Lausanne (EPFL), Switzerland. He completed his $\mathrm{PhD}$ from Alpen-Adria University, Klagenfurt, Austria. He won different awards such as Ernst Mach-Nachbetreuungsstipendium (EZA) fellowship from OEAD, Austria in 2018, Swiss E'xcellent Scholarship from the Swiss Government in 2015, Overseas Scholarship for Doctoral Studies from HEC, etc. Recently he also won an NRPU project from HEC, Pakistan. He is on the editorial boards of several national and international statistics journals as well. 